Pacific

Journal of

Mathematics

\title{
ANGULAR DISTRIBUTION OF \\ DIAMETERS FOR SPHERES \\ AND RAYS FOR PLANES
}

Nobuhiro InNAmi AND YUya UnEme

Volume 277 No. 1

September 2015 


\title{
ANGULAR DISTRIBUTION OF DIAMETERS FOR SPHERES AND RAYS FOR PLANES
}

\author{
NobUhiro InNAmi AND YUyA UnEme
}

\begin{abstract}
Grove and Shiohama used the critical point theory of a distance function to prove the diameter sphere theorem. In light of the angular distribution of minimizing geodesics, we examine and develop the techniques in its proof to make some diameter sphere theorems and study complete noncompact manifolds, using a generalized Toponogov comparison theorem.
\end{abstract}

\section{Introduction}

Let $M$ be a compact Riemannian $n$-manifold with distance $d(\cdot, \cdot)$ induced from its Riemannian metric. Let $\operatorname{diam}(M)=\max \{d(x, y) \mid x, y \in M\}$ denote its diameter. Grove and Shiohama [1977] have proved that if the sectional curvature of $M$ is greater than or equal to 1 and $\operatorname{diam}(M)>\pi / 2$, then $M$ is homeomorphic to an $n$-sphere, using the critical point theory of a distance function. From this point of view, the unit sphere has nice properties as a reference surface. We examine those properties to make some other diameter sphere theorems and show some conditions under which $M$ is diffeomorphic to an $n$-plane. In order to do this, we introduce the angular distribution of minimizing geodesic segments and the reference map from $M$ into a reference surface. The angular distribution measures how the minimizing geodesics are distributed in $M$. The reference map will be used to compare the geometry on $M$ with the geometry on a reference surface $\widetilde{M}$ through the generalized Toponogov comparison theorem.

In Section 2, we define the angular distribution of minimizing geodesic segments connecting two points and the reference map $\Phi_{p, q}$ for $q$ in $(M, p)$ with a base point at $p$ into a reference surface $(\tilde{M}, \tilde{p})$ of revolution with vertex $\tilde{p}$. We propose a domain $D(\tilde{p}, \tilde{q}) \subset \tilde{M}$ such that the generalized Toponogov comparison theorem is valid if $\Phi_{p, q}(M) \subset D(\tilde{p}, \tilde{q})$. Using this terminology we state some theorems.

In Section 3, we summarize some properties of geodesics in a surface of revolution and present the generalized Toponogov comparison theorem of the form used in

Innami's research was partially supported by Grant-in-Aid for Scientific Research (C), 22540072. MSC2010: 53C20, 53C22.

Keywords: sphere, diameter, ray, plane. 
this note. In Section 4, we show some properties of the domain $D(\tilde{p}, \tilde{q})$ and give proofs of the theorems stated in Section 2. In Section 5, we study the case that $\widetilde{M}$ is a $\kappa$-plane $M_{\kappa}$ - which is, by definition, a complete simply connected Riemannian surface with constant Gaussian curvature $\kappa$. We have some sphere theorems depending on the relation among the angular distribution of minimizing geodesic segments, the distance between two points, and the Gaussian curvature of a model surface. In Section 6, we discuss the case of noncompact manifolds referred to a $\kappa$-plane with $\kappa<0$.

Klingenberg [1963] was first interested in radial sectional curvature. Some roles of critical point theory have been introduced in [Abresch and Meyer 1997]. A general introduction to the techniques used in this note is found in [Cheeger and Ebin 1975]. There are some generalized Toponogov comparison theorems for radial curvature. But the version used in this note was first proved in [Itokawa et al. 2001; 2003] and developed in [Kondo and Tanaka 2010; Innami et al. 2013a]. As its application, some diameter sphere theorems have been proved in [Kondo 2007; Kondo and Ohta 2007; Lee 2005; Innami et al. 2013b]. The geometry of geodesics on surfaces of revolution has been developed in [Belegradek et al. 2012; Sinclair and Tanaka 2007; Tanaka 1992].

\section{Definitions and statements}

Let $M$ be a complete Riemannian manifold. We introduce a function $\alpha_{p}(x)$ that measures the angular distribution of minimizing geodesic segments from $x$ to $p$. For $p \in M$ let $d_{p}(x)=d(p, x)$ for all $x \in M$. Let $T_{x} M$ denote the tangent space of $M$ at $x$. Let $A_{p}(x)$ be the set of tangent vectors $T(x, p)^{\bullet}(0)$ at $x \neq p$ of all minimizing geodesic segments $T(x, p)$ from $x$ to $p$. The geodesics are supposed to be parameterized by arclength. Let $\beta_{x}(v)=\min \left\{\angle(v, w) \mid w \in A_{p}(x)\right\}$ for $v \in T_{x} M$ and

$$
\alpha_{p}(x)=\max \left\{\beta_{x}(v) \mid v \in T_{x} M\right\} .
$$

Obviously, $\alpha_{p}(x) \leq \pi$ for all $x \in M, x \neq p$. If $x$ is not a cut point of $p$, then $\alpha_{p}(x)=\pi$. We call $\alpha_{p}(x)$ the angular distribution of $A_{p}(x)$ in the unit sphere $S_{x} M$ in $T_{x} M$. We call $x \in M$ a critical point of $d_{p}$ if $\alpha_{p}(x) \leq \pi / 2$. If $p, q \in M$ satisfy $d(p, q)=\operatorname{diam}(M)$, then $q$ is a critical point of $d_{p}$, and $p$ is a critical point of $d_{q}$.

The distribution of critical points of $d_{p}$ depends on the topological and metric structure of $M$. The diameter sphere theorem is based on the following lemma due to Grove and Shiohama [1977].

Lemma 2.1 (basic lemma). Let $M$ be a complete Riemannian manifold and $p \in M$. If there exists no critical point of $d_{p}$ in $M \backslash\{p\}$, then $M$ is diffeomorphic to the Euclidean space $\mathbb{E}^{n}$. If there exists only one critical point $q \in M \backslash\{p\}$ of $d_{p}$ and 
if $\alpha_{p}(q)<\pi / 2$ or $d_{p}(q)=\max \left\{d_{p}(x) \mid x \in M\right\}$, then $M$ is homeomorphic to an n-sphere.

In this note, using the angular distribution, we propose some conditions under which the assumption of Lemma 2.1 is satisfied. In order to do this we use the generalized Toponogov comparison theorem for radial curvature proved in [Itokawa et al. 2003; Innami et al. 2013a; Kondo and Tanaka 2010].

Let $(\widetilde{M}, \tilde{p})$ be a surface of revolution homeomorphic to a sphere or a plane with a geodesic polar coordinate system $(r, \theta)$ around $\tilde{p}$. Its metric is of class $C^{2}$ and given by

$$
d s^{2}=d r^{2}+m(r)^{2} d \theta^{2},
$$

where $m(r)>0,0<r<\ell \leq \infty, \theta \in S^{1}$, and $m:[0, \ell) \rightarrow \mathbb{R}$ satisfies the Jacobi equation

$$
m^{\prime \prime}+\widetilde{K} m=0, \quad m(0)=0, \quad m^{\prime}(0)=1,
$$

and if $\ell<\infty$,

$$
m(\ell)=0, \quad m^{\prime}(\ell)=-1 .
$$

The function $\widetilde{K}$ is called the radial curvature function of $\tilde{M}$.

Let $(M, p)$ be a complete Riemannian manifold with a base point at $p$. A radial plane $\Pi \subset T_{x} M$ at a point $x \in M$ is a plane containing a vector tangent to a minimizing geodesic segment emanating from $p$. A radial sectional curvature $K_{M}(\Pi)$ is a sectional curvature with respect to a radial plane $\Pi$. We say that $(M, p)$ is referred to $(\tilde{M}, \tilde{p})$ if every radial sectional curvature at $x \in M$ is bounded below by $\widetilde{K}(d(p, x))$, namely, $K_{M}(\Pi) \geq \widetilde{K}(d(p, x))$.

Let $(M, p)$ be referred to $(\tilde{M}, \tilde{p})$. If $\ell<\infty$, we then have $d_{p}(x) \leq \ell$ for all $x \in M$, equality holding if and only if $M$ is isometric to the warped product $S^{n-1} \times_{m}[0, \ell]$, where $n=\operatorname{dim} M$ and $S^{n-1}$ is a sphere; see [Itokawa et al. 2001]. From this fact, we may assume that $\max \left\{d_{p}(x) \mid x \in M\right\}<\ell$ if $\ell<\infty$, because our purpose is to study some conditions on $M$ being homeomorphic to a sphere. Thus, we have the point $\tilde{q}=(d(p, q), 0) \in \tilde{M}$ for any point $q \in M$.

Let $\Phi_{p, q}$ denote the reference map from $M$ to the east side $\widetilde{M}^{+}$of the meridian containing $T(\tilde{p}, \tilde{q})$ in $\widetilde{M}$, namely $\widetilde{M}^{+}=\{(r, \theta) \mid 0 \leq r, 0 \leq \theta \leq \pi\}$. By definition, for a point $x \in M$,

$$
d\left(\tilde{p}, \Phi_{p, q}(x)\right)=d(p, x) \quad \text { and } \quad d\left(\tilde{q}, \Phi_{p, q}(x)\right)=d(q, x) .
$$

It is not certain whether or not every point $x \in M$ has a reference point and every geodesic triangle $\triangle(p q x), q, x \in M$, admits the corresponding geodesic triangle $\triangle(\tilde{p} \tilde{q} \tilde{x}), \tilde{q}, \tilde{x} \in \tilde{M}$. This question has been answered affirmatively under a certain condition in [Innami et al. 2013a]. However, we use only a quarter of $\widetilde{M}$ in the critical point theory. More precisely, as the image space of the reference map $\Phi_{p, q}$, 
we define a special domain $D(\tilde{p}, \tilde{q})$ in $\widetilde{M}^{+}$for $\tilde{q}=\left(r_{0}, 0\right) \in \tilde{M}, 0<r_{0}<\ell$. For $\theta \in[0, \pi / 2]$ let

$$
\begin{aligned}
\lambda_{\tilde{q}}(\theta)=\sup \left\{r>0 \mid \angle\left(v_{s},-\frac{\partial}{\partial r}\right)>\right. & \frac{\pi}{2}, v_{s} \in A_{\tilde{q}}\left(z_{s}\right), \\
& \left.\angle\left(w_{s},-\frac{\partial}{\partial r}\right)<\frac{\pi}{2}, w_{s} \in A_{z_{s}}(\tilde{q}), 0 \leq s<r\right\}
\end{aligned}
$$

where $z_{s}=(s, \theta)$, and set

$$
D(\tilde{p}, \tilde{q})=\left\{(r, \theta) \in \tilde{M} \mid 0 \leq r<\lambda_{\tilde{q}}(\theta), 0 \leq \theta<\pi / 2\right\} \cup\{\tilde{p}, \tilde{q}\} .
$$

Obviously, $D(\tilde{p}, \tilde{q}) \supset T(\tilde{p}, \tilde{q})$, since $\angle(\tilde{p} z \tilde{q})=\pi$ for all $z \in T(\tilde{p}, \tilde{q}) \backslash\{\tilde{p}, \tilde{q}\}$. Moreover, as will be shown in Lemma 4.1, there exists no cut point of $\tilde{q}$ in $D(\tilde{p}, \tilde{q})$. Hence, if $\Phi_{p, q}(M) \subset D(\tilde{p}, \tilde{q})$, then the generalized Toponogov comparison theorem is valid for all geodesic triangles $\Delta(p q x)$ and for all $x \in M$.

We define a dominant triangle for $M$ with respect to $p$ and $q$. Let $z \in \tilde{M}$ and $T$ a minimizing geodesic segment with $z \in T$. For an angle $\omega$ let $S=S(z, T, \omega)$ denote the geodesic such that the angle of $S$ with $T$ at $z$ is $\omega$. We make a trilateral with three geodesic segments:

$$
S_{0}=T(\tilde{p}, \tilde{q}), \quad S_{1}=S\left(\tilde{p}, T(\tilde{p}, \tilde{q}), \alpha_{q}(p)\right), \quad S_{2}=S\left(\tilde{q}, T(\tilde{p}, \tilde{q}), \alpha_{p}(q)\right) .
$$

We call the domain $D_{M}$ bounded by $S_{0}, S_{1}$ and $S_{2}$ a dominant domain for $M$ if it exists. The dominant domain $D_{M}$ becomes a triangle if $S_{1}$ and $S_{2}$ intersect. Otherwise, it may not become a triangle. If $S_{0}, S_{1}$ and $S_{2}$ make a triangle, we call it the dominant triangle for $M$, and it is denoted by $\Delta_{M}=\Delta\left(T(\tilde{p}, \tilde{q}), \alpha_{q}(p), \alpha_{p}(q)\right)$.

For a triangle $\Delta$, the triangle domain bounded by $\Delta$ in $\widetilde{M}^{+}$is also denoted by $\Delta$. If the dominant triangle $\Delta_{M}$ exists and the generalized Toponogov comparison theorem is valid for $(M, p)$ referred to $(\tilde{M}, \tilde{p})$, then $\Phi_{p, q}(M) \subset \Delta_{M}$ because of the Alexandrov convexity. The vertex of the dominant triangle $\Delta_{M}$ other than $\tilde{p}$ and $\tilde{q}$ is denoted by $z\left(\Delta_{M}\right)$.

Theorem 2.2. Let $(M, p)$ be a complete Riemannian manifold referred to $(\tilde{M}, \tilde{p})$. Assume that there exists a point $q$ in $M$ such that the dominant triangle $\Delta_{M}=$ $\triangle\left(T(\tilde{p}, \tilde{q}), \alpha_{q}(p), \alpha_{p}(q)\right)$ for $M$ can be made from $p$ and $q$. If $z\left(\Delta_{M}\right) \in D(\tilde{p}, \tilde{q})$, then $M$ is topologically an $n$-sphere.

We have a generalization of the diameter sphere theorem if we impose a certain condition on $\widetilde{M}$; see Lemma 4.3 . We say that $\widetilde{M}$ is without conjugate points in a half if any point $z \in \operatorname{Int}\left(\widetilde{M}^{+}\right)$has no point conjugate to $z$ along any geodesic segment from $z$ contained in $\operatorname{Int}\left(\widetilde{M}^{+}\right)$. Here $\operatorname{Int}\left(\widetilde{M}^{+}\right)$is the interior of $\widetilde{M}^{+}$. Any point in $\operatorname{Int}\left(\widetilde{M}^{+}\right)$has no cut point in $\operatorname{Int}\left(\widetilde{M}^{+}\right)$if and only if $\widetilde{M}$ is without conjugate points in a half. Tanaka [1992] proved that $\widetilde{M}$ is without conjugate points in a half if $\widetilde{M}$ is a von Mangoldt surface of revolution. 
We say that $\tilde{M}$ is without meridian focal points in a quarter if there exists no focal point of the meridian $\{(r, 0) \mid 0 \leq r \leq \ell\}$ in a quarter $\{(r, \theta) \mid 0 \leq r<\ell, 0<\theta<\pi / 2\}$ of $\tilde{M}$. If $\tilde{M}$ is without conjugate points in a half, then it is without meridian focal points in a quarter; see Proposition 3.1. If $\widetilde{M}$ is without meridian focal points in a quarter, then it is without conjugate points in a quarter; see Proposition 3.2.

If $\tilde{M}$ is without meridian focal points in a quarter and $m^{\prime}(r(\tilde{q}))<0$, then $\triangle(T(\tilde{p}, \tilde{q}), \pi / 2, \pi / 2) \subset D(\tilde{p}, \tilde{q})$; see Lemma 4.3. Kondo and Ohta [2007] have proved the following corollary, assuming that $\tilde{M}$ is a von Mangoldt surface of revolution.

Corollary 2.3. Let $(\tilde{M}, \tilde{p})$ be a reference surface homeomorphic to a sphere such that $\tilde{M}$ is without meridian focal points in a quarter. Let $(M, p)$ be a complete Riemannian manifold referred to $(\tilde{M}, \tilde{p})$. If there exists a point $q \in M$ such that $q$ and $p$ are critical points of $d_{p}$ and $d_{q}$, respectively, and if $m^{\prime}\left(d_{p}(q)\right)<0$, then $M$ is homeomorphic to an n-sphere.

When $\ell=\infty$, let $\tilde{\gamma}(t)=(t, 0)$ for $t \in[0, \infty)$. For $\theta \in[0, \pi]$, let $\lambda_{\tilde{\gamma}}(\theta)$ denote the supremum of those $r>0$ such that there exists a unique coray from $(s, \theta)$, $0<s<r$, to $\tilde{\gamma}$ whose initial tangent vector $v$ satisfies $\angle(v,-\partial / \partial r)>\pi / 2$. Using this function $\lambda_{\tilde{\gamma}}(\theta)$, we define a special domain $D(\tilde{\gamma})$ in a reference surface of revolution $\tilde{M}$. Namely, we set

$$
D(\tilde{\gamma})=\left\{(r, \theta) \in \tilde{M} \mid 0 \leq r<\lambda_{\tilde{\gamma}}(\theta), 0 \leq \theta \leq \pi\right\} .
$$

Obviously, $\lambda_{\tilde{\gamma}}(0)=\infty$. Let $\rho_{\tilde{p}}(\tilde{\gamma})=\sup \left\{\theta_{0} \mid \lambda_{\tilde{\gamma}}(\theta)=\infty\right.$ for $\left.\theta \in\left[0, \theta_{0}\right)\right\}$. When $\tilde{M}$ is a $\kappa$-plane with $\kappa \leq 0$, we have $\rho_{\tilde{p}}(\tilde{\gamma})=0$ if $\kappa<0$ and $\rho_{\tilde{p}}(\tilde{\gamma})=\pi / 2$ if $\kappa=0$. If $\tilde{M}$ is a paraboloid of revolution, then $\rho_{\tilde{p}}(\tilde{\gamma})=\pi$.

Let $\Gamma_{p}$ denote the set of all rays from $p$ in $(M, p)$. Let

$$
\eta_{p}(v)=\min \left\{\angle(v, \dot{\gamma}(0)) \mid \gamma \in \Gamma_{p}\right\}
$$

for any $v \in T_{p} M$, and set

$$
\zeta_{p}=\max \left\{\eta_{p}(v) \mid v \in T_{p} M\right\} .
$$

Obviously, $\zeta_{p} \leq \pi$ for all $p \in M$. We call $\zeta_{p}$ the angular distribution of rays from $p$. We call $\widetilde{M}^{+}\left(\theta_{0}\right)=\left\{(r, \theta) \mid 0 \leq r<\ell, 0 \leq \theta \leq \theta_{0}\right\}$ a sector of $\tilde{M}$ for $\theta_{0} \in[0, \pi]$.

Theorem 2.4. Let $(M, p)$ be a complete noncompact Riemannian n-manifold referred to $(\tilde{M}, \tilde{p})$ such that $\rho_{\tilde{p}}(\tilde{\gamma})>0$. Assume that the sector $\operatorname{Int}\left(\tilde{M}^{+}\left(\rho_{\tilde{p}}(\tilde{\gamma})\right)\right)$ is without conjugate points. If $\zeta_{p}<\rho_{\tilde{p}}(\tilde{\gamma})$, then $M$ is diffeomorphic to an $n$-plane.

Since $\rho_{\tilde{p}}(\tilde{\gamma})=0$ for $M_{\kappa}$ with $\kappa<0$, the theorem shows an advantage of using a surface of revolution as a reference surface. 


\section{Preliminaries}

Let $(\tilde{M}, \tilde{p})$ be a surface of revolution with vertex $\tilde{p}$ and let $\gamma:(-\infty, \infty) \rightarrow \tilde{M}$ be a geodesic with unit speed. We write $\gamma(s)=(r(s), \theta(s))$ for all $s \in(-\infty, \infty)$. Let $\left\{E_{1}(s)=\dot{\gamma}(s), E_{2}(s)\right\}$ denote a set of parallel orthonormal vector fields along $\gamma$. Since the vector field $Y(s)=\partial / \partial \theta$ along $\gamma$ is generated from a variation through geodesics $\gamma_{u}(s)=(r(s), \theta(s)+u)$, it is a Jacobi vector field along $\gamma$. If $\varphi(s)$ denotes the angle of $Y(s)$ with $\dot{\gamma}(s)$, we then have $\left\langle E_{1}(s), Y(s)\right\rangle=m(r(s)) \cos \varphi(s)=v$ which is called the Clairaut relation. Note that $-m(r(0)) \leq v \leq m(r(0))$. The orthogonal complement of $Y(s)$ to $\dot{\gamma}(s)$ is $\sqrt{m(r(s))^{2}-v^{2}} E_{2}(s)$. Therefore,

$$
y(s)=\sqrt{m(r(s))^{2}-v^{2}}
$$

satisfies the Jacobi equation,

$$
y^{\prime \prime}(s)+\widetilde{K}(r(s)) y(s)=0 .
$$

If $C(\gamma)=\left\{s \mid r^{\prime}(s)=0\right\}$, then the number of elements of $C(\gamma)$ is 1 or $\infty$. The Sturm separation theorem states that if $C(\gamma)=\left\{s_{0}\right\}$, then for every $s<s_{0}$ there exists at most one point $\gamma\left(s_{1}\right), s_{1}>s_{0}$, conjugate to $\gamma(s)$. The Clairaut relation states that if $\cdots<s_{-1}<s_{0}<s_{1}<\cdots$ are the solutions of the equation $y(s)=0$, then $\gamma$ is tangent to the parallel circle $r=r\left(s_{i}\right)$ with $m\left(r\left(s_{i}\right)\right)=v$ and $\gamma\left(s_{i}\right)$ are conjugate to one another for $i \in \mathbb{Z}$. From the Sturm separation theorem, if $\bar{y}(s)$ is the length of a perpendicular Jacobi vector field along $\gamma$ such that $\bar{y}\left(t_{0}\right)=0, s_{0}<t_{0}<s_{1}$, then the zeros of $\bar{y}(s)$ appear in each interval $\left(s_{i}, s_{i+1}\right)$ once for every $i \in \mathbb{Z}$.

Proposition 3.1. Let $(\tilde{M}, \tilde{p})$ be a surface of revolution with vertex $\tilde{p}$. If $\tilde{M}$ is without conjugate points in a half, then $\widetilde{M}$ is without meridian focal points in a quarter.

Proof. Suppose that $\tilde{M}$ is not without meridian focal points in a quarter. Then there exists a geodesic $\gamma:[0, a] \rightarrow \operatorname{Int}\left(\tilde{M}^{+}\right)$normal to the meridian $\theta=\pi / 2$ such that $\theta(\gamma(a))=\pi / 2$ and $\gamma(0)$ is a focal point of $\theta=\pi / 2$ along $\gamma$. Since $\tilde{M}$ is a surface of revolution, $\widetilde{M}$ is symmetric with respect to $\theta=\pi / 2$. From this symmetry, if $\gamma_{e}:[0, \infty) \rightarrow \widetilde{M}$ denotes the extension of $\gamma$, we see that $\gamma_{e}(2 a) \in \operatorname{Int}\left(\tilde{M}^{+}\right)$is a point conjugate to $\gamma_{e}(0)$. Namely, $\widetilde{M}$ is not without conjugate points in a half.

Proposition 3.2. Let $(\tilde{M}, \tilde{p})$ be a surface of revolution with vertex $\tilde{p}$. Assume that $\tilde{M}$ is without meridian focal points in a quarter. Then, $\tilde{M}$ is without conjugate points in a quarter. In particular, there exists a unique geodesic segment in $\widetilde{M}^{+}(\pi / 2)$ connecting any two points in $\widetilde{M}^{+}(\pi / 2)$.

Proof. Suppose that there exists a geodesic segment $\omega:[0, L] \rightarrow \widetilde{M}^{+}(\pi / 2)$ such that $\omega(L)$ is the first point conjugate to $\omega(0)$ along $\omega$. Then, $r(s)=r(\omega(s)), s \in[0, L]$, is not monotone because $\widetilde{M}$ is a surface of revolution without meridian focal points in a quarter. Assume that $r^{\prime}\left(s_{0}\right)=0$ at $s_{0}$ with $0<s_{0}<L$. 
The complete extension of $\omega$ is denoted by the same symbol and its parametrization is changed by $\bar{\omega}(s)=\omega\left(s+s_{0}\right), s \in(-\infty, \infty)$. By the symmetry of $\widetilde{M}$ with respect to the meridian through $\bar{\omega}(0), \bar{\omega}\left(s_{0}\right)$ is a point conjugate to $\bar{\omega}\left(s_{0}-L\right)$. From the Sturm separation theorem, there exists a number $L_{1}>0$ such that $s_{0}-L<-L_{1}<0$ and $\bar{\omega}\left(L_{1}\right)$ is a point conjugate to $\bar{\omega}\left(-L_{1}\right)$ along $\bar{\omega}$. Then, $\bar{\omega}\left(L_{1}\right)$ is a focal point of the meridian through $\bar{\omega}(0)$ along $\bar{\omega}$ and $\left|\theta(\bar{\omega}(0))-\theta\left(\bar{\omega}\left(L_{1}\right)\right)\right|<\pi / 2$. This contradicts that $\tilde{M}$ is without meridian focal points in a quarter.

We prove the second part. If there exist two geodesic segments connecting the same endpoints in $\widetilde{M}^{+}(\pi / 2)$, then they may bounds a biangle domain in $\widetilde{M}^{+}(\pi / 2)$. There exists a minimizing geodesic segment in the biangle domain such that the endpoints are conjugate to each other. This contradicts the first part.

Lemma 3.3. Let $(\tilde{M}, \tilde{p})$ be a surface of revolution with vertex $\tilde{p}$. If $\tilde{M}$ is without meridian focal points in a quarter, then $\operatorname{Int}\left(\widetilde{M}^{+}\right)$is foliated by geodesics perpendicular to the meridian $\theta=\pi / 2$. In particular, if $\widetilde{M}$ is compact, then those geodesics cross the meridian $\theta=0$ at points between the focal points along the meridian $\theta=0$.

Proof. Let $z \in \operatorname{Int}\left(\tilde{M}^{+}\right)$. Since $\tilde{M}$ is without meridian focal points in a quarter, there exists a unique foot $w$ of $z$ on $\theta=\pi / 2$, namely $z \in X=\theta^{-1}(\pi / 2)$ and $d(z, w)=d(z, X)$. This proves the first part of the lemma.

If $\tilde{M}$ is compact, then $\tilde{q}=(\ell, 0)$ is the unique point conjugate to $\tilde{p}=(0,0)$. Hence, there exist focal points to $\theta=\pi / 2$ along $\theta=0$ from $\tilde{p}$ and $\tilde{q}$. Let $(a, 0)$ and $(b, 0)$ be focal points of $\theta=\pi / 2$ along $\theta=0$ from $\tilde{p}$ and $(\ell, 0)$, respectively. We then have $a \leq b$. In fact, if $a>b$, then the geodesics normal to $\theta=\pi / 2$ from points near $\tilde{p}$ and $(\ell, 0)$ meet in $\operatorname{Int}\left(M^{+}\right)$, contradicting the first part. If $a=b$, then all geodesics normal to $\theta=\pi / 2$ pass through $(a, 0)$. If $a<b$, then they pass the interval $([a, b], 0)$, keeping their order.

We review the generalized Toponogov comparison theorem. Let $(M, p)$ be a complete Riemannian manifold referred to $(\tilde{M}, \tilde{p})$. Let $q \in M, q \neq p$. For a point $x \in M$, let $\gamma:[0, a] \rightarrow M$ denote a minimizing geodesic segment such that $\gamma(0)=q$ and $\gamma(a)=x$. As was seen in [Itokawa et al. 2003], if $\Phi_{p, q}(\gamma(s)), s \in[0, a]$, do not intersect the cut locus $\operatorname{Cut}(\tilde{q})$ of $\tilde{q}$ in $\tilde{M}$, then the generalized Toponogov comparison theorem for the base angles is valid. Namely, we have

$$
\angle(\tilde{p} \tilde{q} \tilde{x}) \leq \angle(p q x) \quad \text { and } \quad \angle(\tilde{p} \tilde{x} \tilde{q}) \leq \angle(p x q) .
$$

Let $\alpha:[0, b] \rightarrow M$ be a minimizing geodesic segment such that $\alpha(0)=p$ and $\alpha(b)=x$. As was seen in [Innami et al. 2013a], the generalized Toponogov comparison theorem for the angle at $p$ is valid, under the condition that if $\Phi_{p, q}(\alpha(s))$, $s \in[0, b]$, intersects $\operatorname{Cut}(\tilde{q})$ at $s=s_{0}$, then for any minimizing geodesic segment $T\left(\tilde{q}, \Phi_{p, q}\left(\alpha\left(s_{0}\right)\right)\right)$, there exists a minimizing geodesic segment from $q$ to $\alpha\left(s_{0}\right)$ 
satisfying (1). Namely, we then have

$$
\angle(\tilde{q} \tilde{p} \tilde{x}) \leq \angle(q p x) .
$$

For $p, q, x \in M$, the minimum angle $L^{i}(p q x)$ and maximum one $L^{s}(p q x)$ are defined by

$$
\begin{aligned}
& \angle^{i}(p q x)=\min \left\{\angle(v, w) \mid v \in A_{p}(q), w \in A_{x}(q)\right\}, \\
& \angle^{s}(p q x)=\max \left\{\angle(v, w) \mid v \in A_{p}(q), w \in A_{x}(q)\right\} .
\end{aligned}
$$

It should be noted that there may not exist any triangle $\triangle(p q x)$ with three angles $\angle^{s}(p q x), \angle^{s}(p x q)$, and $\angle^{s}(q p x)$.

In this note, we use the generalized Toponogov comparison theorem of the following form, which is a conclusion of the argument in [Itokawa et al. 2003].

Theorem 3.4. Let $(M, p)$ be a complete Riemannian manifold referred to a surface of revolution $(\tilde{M}, \tilde{p})$. Let $q \in M, q \neq p$. If there exists a star-shaped domain $D$ around $\tilde{q}$ contained in the dominant domain $D_{M}$ such that $\Phi_{p, q}(M) \subset D$, then for all $x \in M$,

$$
\angle(\tilde{p} \tilde{q} \tilde{x}) \leq \angle^{i}(p q x), \quad \angle(\tilde{p} \tilde{x} \tilde{q}) \leq \angle^{i}(p x q), \quad \angle(\tilde{q} \tilde{p} \tilde{x}) \leq \angle^{i}(q p x) .
$$

We say that a domain $D \subset \widetilde{M}^{+}$is star-shaped around $\tilde{q}$ in $\tilde{M}$ if there exists a unique minimizing geodesic segment from $\tilde{q}$ to any point $z \in D$ contained in $D$.

\section{Dominant domains}

Let $(\tilde{M}, \tilde{p})$ be a surface of revolution homeomorphic to a sphere or a plane with a geodesic polar coordinate system $(r, \theta)$ around $\tilde{p}$. Let $\tilde{q}=\left(r_{0}, 0\right) \in \tilde{M}, 0<r_{0}<\ell$.

Lemma 4.1. Let $D(\tilde{p}, \tilde{q})$ be the subset defined before. Then, there is no cut point of $\tilde{q}$ in $D(\tilde{p}, \tilde{q})$, and $D(\tilde{p}, \tilde{q})$ is star-shaped around $\tilde{p}$ and $\tilde{q}$.

Proof. Let $z \in D(\tilde{p}, \tilde{q})$ and let $\gamma:[0, a] \rightarrow \tilde{M}, a=d(\tilde{q}, z)$, a minimizing geodesic segment such that $\gamma(0)=\tilde{q}, \gamma(a)=z, \angle(\dot{\gamma}(0),-\partial / \partial r)<\pi / 2$, and $\angle(\dot{\gamma}(a),-\partial / \partial r)<\pi / 2$. If $r(s)=r(\gamma(s)), s \in[0, a]$, then $r^{\prime}(0)<0$ and $r^{\prime}(a)<0$.

We prove that $\gamma(a)$ is not conjugate to $\gamma(0)$ along it. In order to prove this, it is enough to prove that $r(s)$ is monotone decreasing in $s \in[0, a]$, since $\tilde{M}$ is a surface of revolution. If $r^{\prime}(s) \geq 0$ for some $s \in[0, a]$, then, from $r^{\prime}(a)<0$, there exist at least two parameters $s_{1}$ and $s_{2}$ such that $0<s_{1}<s_{2}<a$ and $r^{\prime}\left(s_{1}\right)=r^{\prime}\left(s_{2}\right)=0$. This implies that $\gamma\left(s_{2}\right)$ is a point conjugate to $\gamma\left(s_{1}\right)$ along $\gamma$, contradicting the fact that $\gamma([0, a])$ is minimizing.

Next, we prove that $z$ is joined to $\tilde{q}$ by a unique minimizing geodesic. Suppose for indirect proof that $\gamma_{1}:[0, a] \rightarrow \widetilde{M}$ is another minimizing geodesic segment satisfying 
the same condition as $\gamma$. Set $\varphi(s)=\angle(\dot{\gamma}(s), \partial / \partial \theta)$ and $\varphi_{1}(s)=\angle\left(\dot{\gamma}_{1}(s), \partial / \partial \theta\right)$ for $s \in[0, a]$. Without loss of generality, $0>\varphi(0)>\varphi_{1}(0)>-\pi / 2$, so

$$
m(r(0)) \cos \varphi(0)>m(r(0)) \cos \varphi_{1}(0) .
$$

From this, the Clairaut relation states that

$$
m(r(a)) \cos \varphi(a)>m(r(a)) \cos \varphi_{1}(a) .
$$

Therefore, we have $0>\varphi(a)>\varphi_{1}(a)>-\pi / 2$. On the other hand, since $z$ is the first meeting point of $\gamma$ and $\gamma_{1}$, the relation between $\varphi(a)$ and $\varphi_{1}(a)$ must be $\varphi(a)<\varphi_{1}(a)$, a contradiction. This implies that $z$ is not a cut point of $\tilde{q}$.

We next prove that $\gamma([0, a]) \subset D(\tilde{p}, \tilde{q})$. If $z=\left(r_{0}, \theta\right)$, then we define $z_{t}=(t, \theta)$ for $t \in\left[0, r_{0}\right]$. We set

$$
t_{0}=\sup \left\{s \mid T\left(z_{t}, \tilde{q}\right) \subset D(\tilde{p}, \tilde{q}) \text { for all } t \in[0, s)\right\} .
$$

From the first variation formula, we see there exists a number $\varepsilon>0$ such that there exists a unique minimizing geodesic segment $T\left(z_{t}, \tilde{q}\right)$ and $z_{t} \in D(\tilde{p}, \tilde{q})$ for every $t \in[0, \varepsilon)$. As seen above, $T\left(z_{t}, \tilde{q}\right) \subset D(\tilde{p}, \tilde{q})$ for all $t \in[0, \varepsilon)$; hence $t_{0}>0$. If $T\left(z_{t_{0}}, \tilde{q}\right)$ is tangent to the parallel circle at $\tilde{q}$, then $t_{0}=\lambda_{\tilde{q}}(\theta)$, contradicting $r_{0}<\lambda_{\tilde{q}}(\theta)$. This is not the case. Otherwise, from the facts seen above, there exists a neighborhood of $T\left(z_{t_{0}}, \tilde{q}\right)$ contained in $D(\tilde{p}, \tilde{q})$. This implies that $t_{0}=r_{0}$.

This lemma makes it possible to use the generalized Toponogov comparison theorem if $\Phi_{p, q}(M) \subset D(\tilde{p}, \tilde{q})$.

Lemma 4.2. Let $(M, p)$ be a complete Riemannian manifold referred to $(\tilde{M}, \tilde{p})$. Assume that there exists a point $q$ in $M$ such that the dominant triangle $\Delta_{M}=$ $\triangle\left(T(\tilde{p}, \tilde{q}), \alpha_{q}(p), \alpha_{p}(q)\right)$ for $M$ can be made from $p$ and $q$. If $z\left(\Delta_{M}\right) \in D(\tilde{p}, \tilde{q})$, then $\Phi_{p, q}(M) \subset \Delta_{M} \subset D(\tilde{p}, \tilde{q})$. In particular, the generalized Toponogov comparison theorem by $\Phi_{p, q}$ for $(M, p)$ referred to $(\tilde{M}, \tilde{p})$ is valid.

Proof. From Lemma 4.1, $D(\tilde{p}, \tilde{q})$ is star-shaped around $\tilde{p}$ and $\tilde{q}$. Therefore, the triangle domain $\Delta_{M}$ satisfies $\Delta_{M} \subset D(\tilde{p}, \tilde{q})$.

We prove that $\Phi_{p, q}(M) \subset \Delta_{M}$. For a sufficiently small $\varepsilon>0$, the generalized Toponogov comparison theorem is valid for all triangles $\triangle(p q x)$ if

$$
d(p, x)+d(q, x)<d(p, q)+\varepsilon ;
$$

see [Itokawa et al. 2003; Innami et al. 2013a; Kondo and Tanaka 2010]. Let $\tilde{x}=\Phi_{p, q}(x)$. Since $\angle(\tilde{p} \tilde{q} \tilde{x}) \leq \angle(p q x) \leq \alpha_{p}(q)$ and $\angle(\tilde{q} \tilde{p} \tilde{x}) \leq \angle(q p x) \leq \alpha_{q}(p)$, we have $\tilde{x} \in \Delta_{M}$.

Let $x \in M$ be any point and $\gamma:[0, a] \rightarrow M$, a minimizing geodesic segment such that $\gamma(0)=q$ and $\gamma(a)=x$. We define

$$
t_{0}=\sup \left\{t \mid \Phi_{p, q}(\gamma(s)) \text { is defined and } \Phi_{p, q}(\gamma(s)) \in \Delta_{M} \text { for } s \in[0, t)\right\} .
$$


As is seen above, we have $t_{0}>0$. Suppose for indirect proof that $t_{0}<a$. Then $\tilde{y}=\Phi_{p, q}\left(\gamma\left(t_{0}\right)\right)$ is defined and $\tilde{y} \in T\left(\tilde{q}, z\left(\Delta_{M}\right)\right)$ or $\tilde{y} \in T\left(\tilde{p}, z\left(\Delta_{M}\right)\right)$. Let $\tilde{U}$ be an open set such that $\Delta_{M} \backslash T(\tilde{p}, \tilde{q}) \subset \tilde{U} \subset D(\tilde{p}, \tilde{q})$. Since $\tilde{y}$ is not a cut point of $\tilde{q}$, there exists a number $t_{1}$ with $t_{1}>t_{0}$, such that the points $\Phi_{p, q}(\gamma(s))$ exist in $\tilde{U}$ for all $s \in\left[t_{0}, t_{1}\right]$ and $\tilde{x}_{1}=\Phi_{p, q}\left(\gamma\left(t_{1}\right)\right) \notin \Delta_{M}$. In fact, we find those reference points because of the method in [Itokawa et al. 2003]. Therefore, we have either $\angle\left(\tilde{p} \tilde{q} \tilde{x}_{1}\right)>\alpha_{p}(q)$ or $\angle\left(\tilde{q} \tilde{p} \tilde{x}_{1}\right)>\alpha_{q}(p)$.

On the other hand, since there is no cut point of $\tilde{q}$ in $\widetilde{U}$, the generalized Toponogov comparison theorem is valid in $\Phi_{p, q}^{-1}(\widetilde{U})$. Hence,

$$
\angle\left(\tilde{p} \tilde{q} \tilde{x}_{1}\right) \leq \angle\left(p q \gamma\left(t_{1}\right)\right) \leq \alpha_{p}(q), \quad \angle\left(\tilde{q} \tilde{p} \tilde{x}_{1}\right) \leq \angle\left(q p \gamma\left(t_{1}\right)\right) \leq \alpha_{q}(p),
$$

a contradiction. Therefore, $t_{0}=a$ and $\tilde{x} \in \Delta_{M}$.

Proof of Theorem 2.2. Since $z\left(\Delta_{M}\right) \in D(\tilde{p}, \tilde{q})$, we have both $\alpha_{p}(q)<\pi / 2$ and $\alpha_{q}(p)<\pi / 2$. In particular, $q$ is a critical point of $d_{p}$. In order to apply Lemma 2.1, we have only to prove that there exists no critical point in $M \backslash\{p, q\}$. Let $x \in M$. From Lemma 4.2, the generalized Toponogov comparison theorem by $\Phi_{p, q}$ for $(M, p)$ referred to $(\tilde{M}, \tilde{p})$ is valid. Hence, we have $\pi / 2<\angle(\tilde{p} \tilde{x} \tilde{q}) \leq \angle(p x q)$ since $\tilde{x}=\Phi_{p, q}(x) \in D(\tilde{p}, \tilde{q})$. Consequently, $\alpha_{p}(x)>\pi / 2$, so $x$ is not a critical point of $d_{p}$.

A special case of the next lemma has been proved in [Kondo and Ohta 2007].

Lemma 4.3. Let $(\tilde{M}, \tilde{p})$ be a reference surface without meridian focal points in a quarter and $\tilde{q}=\left(r_{0}, 0\right)$. If $m^{\prime}\left(r_{0}\right)<0$, then $\Delta=\Delta(T(\tilde{p}, \tilde{q}), \pi / 2, \pi / 2) \subset D(\tilde{p}, \tilde{q})$.

Proof. We first prove that the domain $\Omega$ - bounded by the minimizing geodesic segment $T(\tilde{p}, \tilde{q})$, the parallel circle $r=r_{0}=r(\tilde{q})$, and the meridian $\theta=\pi / 2$-is foliated by geodesic segments which are either tangent to $r=r_{0}$ or perpendicular to the meridian $\theta=\pi / 2$ and cross the meridian $\theta=0$.

Let $r_{1}<r_{0}$ satisfy $m\left(r_{1}\right)=m\left(r_{0}\right)$ and $m(r)>m\left(r_{0}\right)$ for all $r \in\left(r_{1}, r_{0}\right)$. Since $m^{\prime}\left(r_{0}\right)<0$, there exists at least one $r_{1}$. The Clairaut relation states that the strip between parallels $r=r_{1}$ and $r=r_{0}$ is foliated by the geodesic segments $T_{\tau}(t)$, $0 \leq t \leq t_{0}$, where $T_{\tau}(0)=\left(r_{0}, \tau\right), \dot{T}_{\tau}(0)=-\left(1 / m\left(r_{0}\right)\right) \partial / \partial \theta$, and $r\left(T_{\tau}(t)\right) \in\left(r_{1}, r_{0}\right)$ for all $t \in\left(0, t_{0}\right)$. Hence the subset $\Omega_{1}$ of $\Omega$ bounded by $T(\tilde{p}, \tilde{q}), r=r_{0}$, and $T_{\pi / 2}$ is foliated by geodesic segments $T_{\tau}$ which are tangent to $r=r_{0}$.

Let $S_{\sigma}(t), \sigma \in\left(0, r_{0}\right)$, denote the geodesic segments such that $S_{\sigma}(0)=(\sigma, \pi / 2)$ and $\dot{S}_{\sigma}(0)=-(1 / m(\sigma)) \partial / \partial \theta$. Since there exists no point focal to $\theta=\pi / 2$ in the sector $\{(r, \theta) \mid \theta \in(0, \pi / 2)\}$, those geodesic segments give a foliation of the subset $\Omega_{2}$ of $\Omega$, bounded by $T(\tilde{p}, \tilde{q}), T_{\pi / 2}$, and $\theta=\pi / 2$; see Lemma 3.3. Since $\Omega=\Omega_{1} \cup \Omega_{2}$, the first claim is proved.

Let $\gamma:[0, L] \rightarrow \widetilde{M}$ denote the geodesic segment which is the edge of $\Delta$ opposite to $\tilde{p}$. Hence, we have $\gamma(0)=\tilde{q}, \dot{\gamma}(0)=\left(1 / m\left(r_{0}\right)\right) \partial / \partial \theta$, and $\theta(\gamma(L))=\pi / 2$. Let 
$z=(r, \pi / 2)$ for $r \in(0, r(\gamma(L)))$. From Proposition 3.2, there exists a unique minimizing geodesic segment $\omega:\left[0, L_{1}\right] \rightarrow \widetilde{M}$ from $\tilde{q}$ to $z$ in $\Delta$.

We have only to prove that the $r$-coordinate of $\omega$ is monotone decreasing. We have $\angle(\dot{\omega}(0),-\partial / \partial r)<\pi / 2$ and $\angle\left(\dot{\omega}\left(L_{1}\right),-\partial / \partial r\right)>\pi / 2$ because of the foliation given in the first part. Therefore, if it is not monotone, then there exist two parameters $s_{1}$ and $s_{2}$ such that $\omega$ is tangent to the parallel circles at $s_{1}$ and $s_{2}$, since then $\omega\left(s_{2}\right)$ is a point conjugate to $\omega\left(s_{1}\right)$, contradicting the fact that $\omega$ is minimizing.

Since the $r$-coordinate of any geodesic segment from $\tilde{q}$ in $\Delta$ is monotone decreasing, $\triangle(T(\tilde{p}, \tilde{q}), \pi / 2, \pi / 2) \subset D(\tilde{p}, \tilde{q})$.

Proof of Corollary 2.3. This corollary follows from Proposition 3.1, Lemma 4.3 and Theorem 2.2, since $\Delta_{M} \subset \Delta(T(\tilde{p}, \tilde{q}), \pi / 2, \pi / 2) \subset D(\tilde{p}, \tilde{q})$.

We need two lemmas to prove Theorem 2.4. For $z \in D(\tilde{\gamma})$, let $z_{t} \in T(\tilde{p}, z)$ be the point such that $r\left(z_{t}\right)=t$.

Lemma 4.4. Let $(\tilde{M}, \tilde{p})$ be a surface of revolution with vertex $\tilde{p}$ such that $\ell=\infty$ and let $\tilde{\gamma}:[0, \infty) \rightarrow \widetilde{M}$ be a ray such that $\tilde{\gamma}(t)=(t, 0)$ for all $t \geq 0$. Let $z \in D(\tilde{\gamma})$. Then, there exists a number $R_{0}>0$ such that the angles of $T\left(z_{t}, \tilde{\gamma}(s)\right)$ with $-\partial / \partial r$ at $z_{t}$ are greater than $\pi / 2$ for all $z_{t} \in T(\tilde{p}, z)$ and $s>R_{0}$.

Proof. For any $s>0$, let $\psi(s)$ be the supremum of the angles of $T\left(z_{t}, \tilde{\gamma}(s)\right)$ with $-\partial / \partial r$ at $z_{t}$ for all $z_{t} \in T(\tilde{p}, z)$. Then $\psi(s)$ is monotone and increasing in $s \in(0, \infty)$, since $(\tilde{M}, \tilde{p})$ is a surface of revolution homeomorphic to a plane. Since $T\left(z_{t}, \tilde{\gamma}(s)\right)$ converges to the corays from $z_{t}$ to $\tilde{\gamma}, \psi(s)$ converges to a real number greater than $\pi / 2$ as $s \rightarrow \infty$.

Lemma 4.5. Let $(M, p)$ be a complete noncompact Riemannian n-manifold referred to $(\tilde{M}, \tilde{p})$. Let $\gamma:[0, \infty) \rightarrow M$ be a ray such that $\gamma(0)=p$. Then, for any points $x \in M$ and $z \in \tilde{M}$, there exists a sequence of parameters $s_{j}$ such that $s_{j} \rightarrow \infty$ and the angles of $T\left(\gamma\left(s_{j}\right), x\right)$ with $-\dot{\gamma}\left(s_{j}\right)$ and $T\left(\tilde{\gamma}\left(s_{j}\right), z\right)$ with $-\dot{\tilde{\gamma}}\left(s_{j}\right)$ converge to zero as $j \rightarrow \infty$.

Proof. This follows from the following inequality and the first variation formula.

$$
|2 s-d(\gamma(s), x)-d(\tilde{\gamma}(s), z)| \leq d(\gamma(0), x)+d(\tilde{\gamma}(0), z) .
$$

In fact, if this lemma is not true, then the left hand side of the inequality goes to $\infty$ as $s \rightarrow \infty$.

Proof of Theorem 2.4. From Lemma 2.1, we have only to prove that there exists no critical point of $d_{p}$ in $M \backslash\{p\}$. Let $x \in M \backslash\{p\}$ and $\alpha:[0, a] \rightarrow M$ a minimizing geodesic segment such that $\alpha(0)=p$ and $\alpha(a)=x$. From the assumption, there exists a ray $\gamma:[0, \infty) \rightarrow M$ from $p$ such that $\angle(\dot{\gamma}(0), \dot{\alpha}(0)) \leq \zeta_{p}$. Let $z=$ $(d(p, x), \xi)$, where $\zeta_{p}<\xi<\rho_{\tilde{p}}(\tilde{\gamma})$. For this point $z$, let $R_{0}>0$ denote the number given in Lemma 4.4. Furthermore, for this $x$ and $z$, there exists a number $s_{0}>R_{0}$ 
satisfying the property in Lemma 4.5. If $\Delta$ is the triangle domain bounded by $T\left(\tilde{p}, \tilde{\gamma}\left(s_{0}\right)\right) \cup T\left(\tilde{\gamma}\left(s_{0}\right), z\right) \cup T(\tilde{p}, z)$, as is seen in the proof of Lemma 4.1, then $\Delta \subset D\left(\tilde{p}, \tilde{\gamma}\left(s_{0}\right)\right)$.

We have to prove that $\Phi_{p, \gamma\left(s_{0}\right)}(x) \in \Delta$. Since $\tilde{p}$ is not a cut point of $\tilde{\gamma}\left(s_{0}\right)$, there exists a number $\varepsilon>0$ such that if $0 \leq t<\varepsilon$, then $y_{t}=\Phi_{p, \gamma\left(s_{0}\right)}(\alpha(t)) \in \Delta$. In fact, $r\left(y_{t}\right)=t$ and $\angle\left(\tilde{\gamma}\left(s_{0}\right) \tilde{p} y_{t}\right) \leq \angle\left(\gamma\left(s_{0}\right) p x\right)<\xi$, since the generalized Toponogov comparison theorem is valid in some neighborhood of $\gamma\left(\left[0, s_{0}\right]\right)$. Set

$$
t_{0}=\sup \left\{t \in(0, a] \mid y_{t} \in \Delta\right\} .
$$

As seen before, $t_{0}>0$ and $\alpha\left(t_{0}\right) \in \Delta$. If $t_{0} \neq a$, we find a number $\varepsilon_{1}>0$ such that $y_{t} \in \Delta$ for all $t \in\left(t_{0}, t_{0}+\varepsilon_{1}\right)$, since the sector $\operatorname{Int}\left(\widetilde{M}^{+}\left(\rho_{\tilde{p}}(\tilde{\gamma})\right)\right)$ is without conjugate points and, hence, the generalized Toponogov comparison theorem is valid. This contradicts the choice of $t_{0}$. Thus, we have $y_{a}=\Phi_{p, \gamma\left(s_{0}\right)}(x) \in \Delta$.

Therefore, $\angle\left(\gamma\left(s_{0}\right) x p\right) \geq \angle\left(\tilde{\gamma}\left(s_{0}\right) y_{a} \tilde{p}\right)>\pi / 2$, meaning that $\alpha_{p}(x)>\pi / 2$. Thus, $x$ is not a critical point of $d_{p}$.

\section{The $\kappa$-plane as a reference surface for spheres}

Let $M_{\kappa}$ be the $\kappa$-plane, by definition isometric to the 2-sphere $S^{2}(1 / \sqrt{\kappa})$ with radius $1 / \sqrt{\kappa}$ if $\kappa>0$, the Euclidean plane $\mathbb{E}^{2}$ if $\kappa=0$, or the Poincaré disk with Gauss curvature $\kappa$ if $\kappa<0$. Notice that $M_{\kappa}$ is without meridian focal points in a quarter. However, Lemma 4.3 is not applied if $\kappa \leq 0$, since no parameter $r_{0}$ exists such that $m^{\prime}\left(r_{0}\right)<0$. This means that the condition of being critical, namely $\alpha_{p}(q) \leq \pi / 2$ and $\alpha_{q}(p) \leq \pi / 2$, are not enough for a sphere theorem if the reference surface is $M_{\kappa}, \kappa \leq 0$. We need a restricted condition on $\alpha_{p}(q)$ and $\alpha_{q}(p)$ which depends on the distance $d(p, q)$ and $\kappa$.

Let $M$ be a complete Riemannian $n$-manifold with sectional curvature bounded below by a constant $\kappa$. For points $p, q \in M$ we have points $\tilde{p}, \tilde{q} \in M_{\kappa}$ such that $d(p, q)=d(\tilde{p}, \tilde{q})$. When $\kappa>0$, we assume that $d(p, q)<\pi / \sqrt{\kappa}$. Because, in general, $d(p, q) \leq \pi / \sqrt{\kappa}$, with equality holding if and only if $M$ is isometric to the sphere with radius $1 / \sqrt{\kappa}$.

Obviously, $D(\tilde{p}, \tilde{q})=\left\{z \in M_{\kappa} \mid \angle(\tilde{p} z \tilde{q})>\pi / 2\right\}$. More precisely, $z \in D(\tilde{p}, \tilde{q})$ if and only if $z$ satisfies the inequalities:

(1) $\cos \sqrt{\kappa} d(\tilde{p}, \tilde{q})<\cos \sqrt{\kappa} d(\tilde{p}, z) \cos \sqrt{\kappa} d(\tilde{q}, z) \quad$ if $\kappa>0$,

(2) $d(\tilde{p}, \tilde{q})^{2}>d(\tilde{p}, z)^{2}+d(\tilde{q}, z)^{2} \quad$ if $\kappa=0$,

(3) $\cosh \sqrt{-\kappa} d(\tilde{p}, \tilde{q})>\cosh \sqrt{-\kappa} d(\tilde{p}, z) \cosh \sqrt{-\kappa} d(\tilde{q}, z) \quad$ if $\kappa<0$.

Example 5.1. In $M_{1}$, if $\tilde{p}$ and $\tilde{q}$ satisfy $\pi>d(\tilde{p}, \tilde{q})>\pi / 2$ and $z \in M_{1}$ is a meeting point of the perpendiculars to $T(\tilde{p}, \tilde{q})$ at $\tilde{p}$ and $\tilde{q}$, then the domain bounded by 
the geodesic triangle $\triangle(\tilde{p} z \tilde{q})$ is contained in $D(\tilde{p}, \tilde{q})$. In $M_{0}=\mathbb{E}^{2}$, by elementary geometry, we see that $D(\tilde{p}, \tilde{q})$ is the open disk with diameter $d(\tilde{p}, \tilde{q})$.

Corollary 5.2. Let $M$ be a complete Riemannian manifold with sectional curvature bounded below by $\kappa$. Assume that there exist two points $p$ and $q$ such that a dominant triangle $\Delta_{M}=\triangle\left(T(\tilde{p}, \tilde{q}), \alpha_{q}(p), \alpha_{q}(p)\right)$ for $M$ can be made from $p$ and $q$. If its inner angle at $z\left(\Delta_{M}\right)$ is greater than $\pi / 2$, then $M$ is topologically an n-sphere.

Proof. Since the dominant triangle $\Delta_{M}$ is contained in $D(\tilde{p}, \tilde{q})$, this proposition follows from Theorem 2.2.

Let $\tilde{p}, \tilde{q} \in M_{\kappa}$ such that $\tilde{p} \neq \tilde{q}$. Let $E(\tilde{p}, \tilde{q})=\left\{z \in M_{\kappa} \mid \angle(\tilde{p} z \tilde{q})=\pi / 2\right\}$. Namely, $E(\tilde{p}, \tilde{q})=\partial D(\tilde{p}, \tilde{q})$. Set

$$
\omega=\omega(\kappa, d(\tilde{p}, \tilde{q}))=\min \{\angle(z \tilde{p} \tilde{q})+\angle(z \tilde{q} \tilde{p}) \mid z \in E(\tilde{p}, \tilde{q})\} .
$$

Obviously, $\omega>0$. From the Gauss-Bonnet formula, we have $\omega=\pi / 2$ when $\kappa \geq 0$ and $\omega<\pi / 2$ when $\kappa<0$. If $\alpha_{p}(q)+\alpha_{q}(p)<\omega$, then there exists a dominant triangle for $M$.

Corollary 5.3. Let $M$ be a complete Riemannian n-manifold with sectional curvature bounded below by $\kappa$. If there exist two points $p, q \in M$ such that

$$
\alpha_{p}(q)+\alpha_{q}(p)<\omega(\kappa, d(p, q))
$$

then $M$ is homeomorphic to an $n$-sphere.

Proof. From the assumption, there exists a dominant triangle $\Delta_{M}$ for $M$ which is contained in $D(\tilde{p}, \tilde{q})$. This corollary follows from Theorem 2.2.

Remark 5.4. Let $\mathbb{E}^{2}$ denote the Euclidean plane. Let $G$ be the isometry group generated by two translations $\mu(x, y)=(x+a, y)$ and $v(x, y)=(x, y+b)$ where $a$ and $b$ are positive constants. The quotient space is a flat torus $T^{2}=\mathbb{E}^{2} / G$. The equivalence class containing $(x, y)$ is written with $[(x, y)]$. Let $p=[(a / 2, b / 2)]$ and $q=[(0,0)]$. There exist four minimizing geodesic segments connecting $p$ and $q$ in $T^{2}$. We then have $d(p, q)=\operatorname{diam}\left(T^{2}\right)$ and $\alpha_{p}(q)+\alpha_{q}(p)=\pi / 2$, meaning that Corollary 5.3 is optimal.

Let $\mathcal{C}=\mathcal{C}(p, q)$ be the set of all midpoints between $p$ and $q$, namely

$$
\mathcal{C}=\{x \in M \mid d(p, x)=d(x, q)=d(p, q) / 2\} .
$$

If $x \in \mathcal{C}$, then $T(p, x) \cup T(x, q)$ is the unique minimizing geodesic segment through $x$ connecting $p$ and $q$.

Corollary 5.5. Let $M$ be a complete Riemannian n-manifold of nonnegative sectional curvature and $p, q \in M$. If $d(x, \mathcal{C}(p, q))<d(p, q) / 2$ for all $x \in M \backslash\{p, q\}$, then $M$ is topologically an $n$-sphere. 
Proof. We have only to prove that any point $x \in M \backslash\{p, q\}$ is not a critical point of the distance function $d_{p}$. We use the Euclidean plane $\mathbb{E}^{2}$ as a model space for the Toponogov comparison theorem. Let $\widetilde{T}=T(\tilde{p}, \tilde{q})$ be a segment in $\mathbb{E}^{2}$ with length $d(p, q)$ and $\tilde{m}$ the midpoint of $\widetilde{T}$.

Let $x \in M \backslash\{p, q\}$. From the assumption, there exists a midpoint $m$ between $p$ and $q$ such that $d(x, m)<d(p, q) / 2$. Let $\triangle(\tilde{p} \tilde{q} \tilde{x})$ be the comparison triangle in $\mathbb{E}^{2}$ corresponding to $\triangle(p q x)$. Then it follows from the Alexandrov convexity that $d(x, m) \geq d(\tilde{x}, \tilde{m})$. Therefore, we have $d(\tilde{m}, \tilde{x})<d(\tilde{p}, \tilde{q}) / 2$. Thus we have $\angle(\tilde{p} \tilde{x} \tilde{q})>\pi / 2$. From the Toponogov comparison theorem, we have $\angle(p x q)>\pi / 2$. This implies that $x$ is not a critical point of $d_{p}$.

Remark 5.6. Let $T^{2}, p$, and $q$ be as in Remark 5.4. Let $s=[(0, b / 2)]$. We then have $d(s, x)=\operatorname{diam}\left(T^{2}\right) / 2$ for all $x \in \mathcal{C}(p, q)$. From this example, Corollary 5.5 is optimal.

\section{Noncompact manifolds referred to $M_{\kappa}$}

Let $M$ be a complete noncompact Riemannian $n$-manifold with sectional curvature bounded below by $\kappa \leq 0$ and $M_{\kappa}$ the $\kappa$-plane. Let $\gamma$ be a ray in $M$ with $\gamma(0)=p$. The Busemann function $f_{\gamma}$ for $\gamma$ is defined by

$$
f_{\gamma}(x)=\lim _{t \rightarrow \infty}(t-d(x, \gamma(t))), \quad x \in M .
$$

Let $B_{\gamma}(x)$ be the open horoball of a ray $\gamma$ given by $\left\{y \in M \mid f_{\gamma}(y)>f_{\gamma}(x)\right\}$.

Let $\Gamma_{p}$ denote the set of all rays from $p$ in $M$. The super Busemann function $f_{p}$ is given by $f_{p}(x)=\sup _{\gamma \in \Gamma_{p}} f_{\gamma}(x)$ for all $x \in M$.

Let $\tilde{\gamma}$ be a fixed ray in $M_{\kappa}$ with $\tilde{\gamma}(0)=\tilde{p}$. We call $B_{\tilde{\gamma}}(z)$ a horoball of $\tilde{\gamma}$ determined by $z \in M_{\kappa}$. Since $\kappa \leq 0$, all horoballs are convex in $M_{\kappa}$, meaning that if $w_{1}, w_{2} \in B_{\tilde{\gamma}}(z)$, then the unique minimizing geodesic segment $T\left(z_{1}, z_{2}\right)$ is contained in $B_{\tilde{\gamma}}(z)$.

Let $v(z)$ be the unit tangent vector at $z \in M_{\kappa}$ of the coray to $\tilde{\gamma}$ and $w(z)$ the unit tangent vector of geodesic segment from $z$ to $\tilde{p}$ at $z$, respectively. Set

$$
D(\tilde{\gamma})=\left\{z \in M_{\kappa} \mid \angle(v(z), w(z))>\pi / 2\right\} .
$$

We have $D(\tilde{\gamma})=\lim _{t \rightarrow \infty} B_{\tilde{\gamma}(t)}(t)$ if $\kappa=0$. When $\kappa<0$, the boundary $\partial D(\tilde{\gamma})$ of $D(\tilde{\gamma})$ is the trace of those points $z(t) \in M_{\kappa}, t \geq 0$, such that the straight line tangent to the horocircle $f_{\tilde{\gamma}}^{-1}(t)$ through $\tilde{\gamma}(t)$ at $z(t)$ passes through $\tilde{p}$.

Example 6.1. Let $M_{-1}=\left\{(x, y) \mid x^{2}+y^{2}<1\right\}$ and

$$
d s^{2}=\frac{4\left(d x^{2}+d y^{2}\right)}{\left(1-x^{2}-y^{2}\right)^{2}}
$$


be the Poincaré disk model. Let $\tilde{p}=(0,0)$ and $\tilde{\gamma}([0, \infty))=\{(0, t) \mid 0 \leq t<1\}$. If $x=r \cos \theta, y=r \sin \theta$, then $\partial D(\tilde{\gamma})$ is the trace of the curve given by the equation $r=\tan (\theta / 2), 0<\theta<\pi / 2$. In fact, since any horocircle of $\tilde{\gamma}$ is a subarc of a circle with center $(u \cos \theta, 1)$ and radius $u \cos \theta$ and any geodesic from $(0,0)$ is a subsegment of a straight line through $(0,0)$ with slope $\tan \theta$, they meet at points satisfying

$$
r=u-u \cos \theta, \quad 1=u \sin \theta .
$$

Hence, we have

$$
r=\frac{1-\cos \theta}{\sin \theta}=\frac{2 \sin ^{2}(\theta / 2)}{2 \sin (\theta / 2) \cos (\theta / 2)}=\frac{\sin (\theta / 2)}{\cos (\theta / 2)}
$$

Here we assume that $\kappa<0$. As before, let $z(t)=\partial D(\tilde{\gamma}) \cap f_{\tilde{\gamma}}^{-1}(t)$ in $M_{\kappa}$. Let $\rho_{\tilde{p}}(t)$ be the angle of $\tilde{\gamma}$ with $T(\tilde{p}, z(t))$ at $\tilde{p}$ for $t \geq 0$. Then $\rho_{\tilde{p}}(0)=\pi / 2$ and $\lim _{t \rightarrow \infty} \rho_{\tilde{p}}(t)=0$. Moreover, $\rho_{\tilde{p}}(t)$ is monotone decreasing in $t \geq 0$.

Let $\tilde{\gamma}$ be a fixed ray in $\left(M_{\kappa}, \tilde{p}\right)$ with $\tilde{\gamma}(0)=\tilde{p}$. Let $\Psi_{p}$ be the reference map from $M$ to $M_{\kappa}{ }^{+}$. By definition, we have, for all points $x \in M$,

$$
d\left(\tilde{p}, \Psi_{p}(x)\right)=d(p, x), \quad f_{\tilde{\gamma}}\left(\Psi_{p}(x)\right)=f_{p}(x) .
$$

Corollary 6.2. Let $M$ be a complete noncompact Riemannian n-manifold with sectional curvature bounded below by $\kappa$. If there exists a point $p \in M$ such that $\Psi_{p}(M \backslash\{p\}) \subset D(\tilde{\gamma})$, then $M$ is diffeomorphic to the Euclidean space $\mathbb{E}^{n}$.

Proof. From the definition of $D(\tilde{\gamma})$, there exists no critical point of $d_{p}$ in $M \backslash\{p\}$. Lemma 2.1 proves this corollary.

Proposition 6.3. Let $M$ denote a complete noncompact Riemannian n-manifold with sectional curvature bounded below by $\kappa<0$. Assume that $\zeta_{p}<\pi / 2$. Then $p$ is a minimum point of $f_{p}$ in $M$. If $t_{0}$ satisfies $\rho_{\tilde{p}}\left(t_{0}\right)=\zeta_{p}$, then there exists no critical point of $d_{p}$ in $f_{p}^{-1}\left(\left(0, t_{0}\right)\right)$.

Proof. Since $\zeta_{p}<\pi / 2$, it follows that $f_{p}(p)=0$ is a minimum of $f_{p}$ in $M$. Let $x \in M$ be such that $0<f_{p}(x)<t_{0}$. Let $v$ be the initial tangent vector of a minimizing geodesic segment from $p$ to $x$. From the definition of $\zeta_{p}$, there exists $\gamma \in \Gamma_{p}$ such that $\angle(v, \dot{\gamma}(0)) \leq \zeta_{p}$. From the definition of $f_{p}$, we have $f_{\gamma}(x) \leq f_{p}(x)<t_{0}$ and, hence, from the Toponogov comparison theorem,

$$
\rho_{\tilde{p}}\left(f_{\gamma}(x)\right)>\rho_{\tilde{p}}\left(t_{0}\right)=\zeta_{p} \geq \angle(v, \dot{\gamma}(0)) \geq \angle(\tilde{v}, \dot{\tilde{\gamma}})
$$

where $\tilde{v}$ is the initial tangent vector of the minimizing geodesic segment from $\tilde{p}$ to $\Psi_{\gamma}(x)$ in $M_{\kappa}$. This inequality shows $\Psi_{\gamma}(x) \in D(\tilde{\gamma})$. 


\section{References}

[Abresch and Meyer 1997] U. Abresch and W. T. Meyer, "Injectivity radius estimates and sphere theorems", pp. 1-47 in Comparison geometry (Berkeley, CA, 1993-1994), edited by K. Grove and P. Petersen, Mathematical Sciences Research Institute Publications 30, Cambridge University Press, 1997. MR 98e:53052 Zbl 0888.53001

[Belegradek et al. 2012] I. Belegradek, E. Choi, and N. Innami, "Rays and souls in von Mangoldt planes”, Pacific J. Math. 259:2 (2012), 279-306. MR 2988492 Zbl 1268.53041

[Cheeger and Ebin 1975] J. Cheeger and D. G. Ebin, Comparison theorems in Riemannian geometry, North-Holland Mathematical Library 9, North-Holland, Amsterdam, 1975. Reprinted by AMS Chelsea, Providence, RI, 2008. MR 0458335 Zbl 0309.53035

[Grove and Shiohama 1977] K. Grove and K. Shiohama, "A generalized sphere theorem", Ann. of Math. (2) 106:2 (1977), 201-211. MR 58 \#18268 Zbl 0341.53029

[Innami et al. 2013a] N. Innami, K. Shiohama, and Y. Uneme, "The Alexandrov-Toponogov comparison theorem for radial curvature", Nihonkai Math. J. 24:2 (2013), 57-91. MR 3178500 Zbl 1296.53067

[Innami et al. 2013b] N. Innami, K. Shiohama, and Y. Uneme, "A sphere theorem for radial curvature", Nihonkai Math. J. 24:2 (2013), 93-102. MR 3178501 Zbl 1288.53027

[Itokawa et al. 2001] Y. Itokawa, Y. Machigashira, and K. Shiohama, "Maximal diameter theorems for manifolds with restricted radial curvature", pp. 61-68 in Proceedings of the 5th Pacific Rim Geometry Conference (Sendai, 2000), edited by S. Nishikawa, Tôhoku Mathematical Publications 20, Tôhoku University, Sendai, 2001. MR 2002k:53055 Zbl 1065.53033

[Itokawa et al. 2003] Y. Itokawa, Y. Machigashira, and K. Shiohama, "Generalized Toponogov's theorem for manifolds with radial curvature bounded below", pp. 121-130 in Explorations in complex and Riemannian geometry, edited by J. Bland et al., Contemporary Mathematics 332, American Mathematical Society, Providence, RI, 2003. MR 2004j:53051 Zbl 1046.53017

[Klingenberg 1963] W. Klingenberg, "Manifolds with restricted conjugate locus", Ann. of Math. (2) 78 (1963), 527-547. MR 28 \#2506 Zbl 0117.38701

[Kondo 2007] K. Kondo, "Radius sphere theorems for compact manifolds with radial curvature bounded below", Tokyo J. Math. 30:2 (2007), 465-475. MR 2009b:53061 Zbl 1145.53025

[Kondo and Ohta 2007] K. Kondo and S.-I. Ohta, "Topology of complete manifolds with radial curvature bounded from below", Geom. Funct. Anal. 17:4 (2007), 1237-1247. MR 2008k:53069 Zbl 1144.53050

[Kondo and Tanaka 2010] K. Kondo and M. Tanaka, "Total curvatures of model surfaces control topology of complete open manifolds with radial curvature bounded below. II", Trans. Amer. Math. Soc. 362:12 (2010), 6293-6324. MR 2011f:53061 Zbl 1225.53034

[Lee 2005] H. Lee, "Generalized Alexandrov-Toponogov theorems for radially curved manifolds and their applications”, Kyushu J. Math. 59:2 (2005), 365-373. MR 2006i:53036 Zbl 1098.53029

[Sinclair and Tanaka 2007] R. Sinclair and M. Tanaka, "The cut locus of a two-sphere of revolution and Toponogov's comparison theorem", Tohoku Math. J. (2) 59:3 (2007), 379-399. MR 2008k:53075 Zbl 1158.53033

[Tanaka 1992] M. Tanaka, "On the cut loci of a von Mangoldt's surface of revolution", J. Math. Soc. Japan 44:4 (1992), 631-641. MR 93h:53034 Zbl 0789.53023

Received March 30, 2014. Revised December 10, 2014. 
NOBUHIRO INNAMI

DEPARTMENT OF MATHEMATICS

FACULTY OF SCIENCE

NIIGATA UNIVERSITY

NIIGATA 950-2181

JAPAN

innami@math.sc.niigata-u.ac.jp

YUYA UNEME

GRaduAte School of Science And TeChnology

NIIGATA UNIVERSITY

NiIGATA 950-2181

JAPAN

f12j004g@alumni.niigata-u.ac.jp 


\title{
PACIFIC JOURNAL OF MATHEMATICS
}

\author{
msp.org/pjm
}

Founded in 1951 by E. F. Beckenbach (1906-1982) and F. Wolf (1904-1989)

\section{EDITORS}

Don Blasius (Managing Editor)

Department of Mathematics

University of California

Los Angeles, CA 90095-1555

blasius@math.ucla.edu

\author{
Paul Balmer \\ Department of Mathematics \\ University of California \\ Los Angeles, CA 90095-1555 \\ balmer@math.ucla.edu \\ Robert Finn \\ Department of Mathematics \\ Stanford University \\ Stanford, CA 94305-2125 \\ finn@math.stanford.edu \\ Sorin Popa \\ Department of Mathematics \\ University of California \\ Los Angeles, CA 90095-1555 \\ popa@math.ucla.edu
}

\author{
Vyjayanthi Chari \\ Department of Mathematics \\ University of California \\ Riverside, CA 92521-0135 \\ chari@math.ucr.edu \\ Kefeng Liu \\ Department of Mathematics \\ University of California \\ Los Angeles, CA 90095-1555 \\ liu@math.ucla.edu \\ Jie Qing \\ Department of Mathematics \\ University of California \\ Santa Cruz, CA 95064 \\ qing@ cats.ucsc.edu
}

\section{PRODUCTION}

Silvio Levy, Scientific Editor, production@msp.org

\section{SUPPORTING INSTITUTIONS}

ACADEMIA SINICA, TAIPEI

CALIFORNIA INST. OF TECHNOLOGY

INST. DE MATEMÁTICA PURA E APLICADA

KEIO UNIVERSITY

MATH. SCIENCES RESEARCH INSTITUTE

NEW MEXICO STATE UNIV.

OREGON STATE UNIV.

\author{
STANFORD UNIVERSITY \\ UNIV. OF BRITISH COLUMBIA \\ UNIV. OF CALIFORNIA, BERKELEY \\ UNIV. OF CALIFORNIA, DAVIS \\ UNIV. OF CALIFORNIA, LOS ANGELES \\ UNIV. OF CALIFORNIA, RIVERSIDE \\ UNIV. OF CALIFORNIA, SAN DIEGO \\ UNIV. OF CALIF., SANTA BARBARA
}

\author{
Daryl Cooper \\ Department of Mathematics \\ University of California \\ Santa Barbara, CA 93106-3080 \\ cooper@math.ucsb.edu \\ Jiang-Hua Lu \\ Department of Mathematics \\ The University of Hong Kong \\ Pokfulam Rd., Hong Kong \\ jhlu@maths.hku.hk \\ Paul Yang \\ Department of Mathematics \\ Princeton University \\ Princeton NJ 08544-1000 \\ yang@math.princeton.edu
}

These supporting institutions contribute to the cost of publication of this Journal, but they are not owners or publishers and have no responsibility for its contents or policies.

See inside back cover or msp.org/pjm for submission instructions.

The subscription price for 2015 is US \$420/year for the electronic version, and \$570/year for print and electronic.

Subscriptions, requests for back issues and changes of subscribers address should be sent to Pacific Journal of Mathematics, P.O. Box 4163, Berkeley, CA 94704-0163, U.S.A. The Pacific Journal of Mathematics is indexed by Mathematical Reviews, Zentralblatt MATH, PASCAL CNRS Index, Referativnyi Zhurnal, Current Mathematical Publications and Web of Knowledge (Science Citation Index).

The Pacific Journal of Mathematics (ISSN 0030-8730) at the University of California, c/o Department of Mathematics, 798 Evans Hall \#3840, Berkeley, CA 94720-3840, is published twelve times a year. Periodical rate postage paid at Berkeley, CA 94704, and additional mailing offices. POSTMASTER: send address changes to Pacific Journal of Mathematics, P.O. Box 4163, Berkeley, CA 94704-0163.

PJM peer review and production are managed by EditFLOW ${ }^{\circledR}$ from Mathematical Sciences Publishers.

\section{PUBLISHED BY}

\section{mathematical sciences publishers \\ nonprofit scientific publishing}

http://msp.org/

(C) 2015 Mathematical Sciences Publishers 


\section{PACIFIC JOURNAL OF MATHEMATICS}

Volume $277 \quad$ No. $1 \quad$ September 2015

Real positivity and approximate identities in Banach algebras

DAVID P. BLECHER and NARUTAKA OZAWA

On shrinking gradient Ricci solitons with nonnegative sectional curvature 61

Mingliang CAI

From quasimodes to resonances: exponentially decaying perturbations

ORAN GANNOT

A general simple relative trace formula

JAYCE R. GETZ and HEEKYOUNG HAHN

Chern-Simons functions on toric Calabi-Yau threefolds and

Donaldson-Thomas theory

\section{ZHENG HUA}

On the flag curvature of a class of Finsler metrics produced by the navigation problem

LiBING HUANG and XIAOHUAN MO

Angular distribution of diameters for spheres and rays for planes

NOBUHIRO INNAMI and YUYA UNEME

A note on an $L^{p}$-Brunn-Minkowski inequality for convex measures in the 187 unconditional case

ARNAUD MARSIGLIETTI

Structure of seeds in generalized cluster algebras

TOMOKI NAKANISHI

Inequalities of Alexandrov-Fenchel type for convex hypersurfaces in hyperbolic space and in the sphere

Yong WeI and ChangWei XiONG

Upper bounds of root discriminant lower bounds 